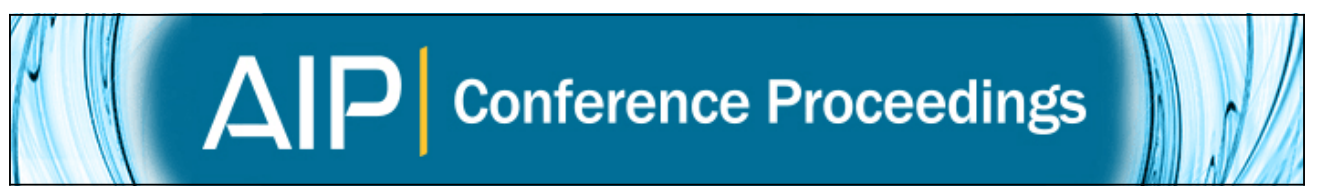

\title{
Collaboration and Competition in Exoplanet Research
}

Charles Beichman

Citation: AIP Conference Proceedings 1158, 373 (2009); doi: 10.1063/1.3215899

View online: http://dx.doi.org/10.1063/1.3215899

View Table of Contents:

http://scitation.aip.org/content/aip/proceeding/aipcp/1158?ver=pdfcov

Published by the AIP Publishing

Articles you may be interested in

Detection of Debris Disks and Wide Orbit Planets with the LBTI

AIP Conf. Proc. 1158, 313 (2009); 10.1063/1.3215875

Nulling Measurements with the Keck Interferometer

AIP Conf. Proc. 1158, 307 (2009); 10.1063/1.3215874

Coronagraphic Techniques for Direct Imaging of Exoplanets and Disks

AIP Conf. Proc. 1158, 285 (2009); 10.1063/1.3215871

Observations of Extrasolar Planets During the non-Cryogenic Spitzer Space Telescope Mission

AIP Conf. Proc. 943, 89 (2007); 10.1063/1.2806789

Radial velocity measurements of a sample of K-giants with the Hobby-Eberly telescope AIP Conf. Proc. 752, 38 (2005); 10.1063/1.1893331 


\title{
Collaboration and Competition in Exoplanet Research
}

\author{
Charles Beichman \\ NASA ExoPlanet Science Institute, Jet Propulsion Laboratory, California Institute of \\ Technology, Pasadena, CA
}

\begin{abstract}
Collaboration and competition are strong driving forces in the modern search for exoplanets. It appears among individuals, agencies and nations, as well as between observing techniques and theoretical interpretation. I will argue that these forces, taken in balance, are beneficial to the field and are partly responsible for the rapid progress in the search for planets and ultimately the search for life beyond the solar system. Specific examples will include indirect detection of Earth analogs from ground and space and the direct detection of gas giant and terrestrial planets.
\end{abstract}

Keywords: Planets, Astrometry, Search for Life, JWST, imaging

PACS: $97.82 .-j$

\section{COMPETITION, COLLABORATION AND COORDINATION}

My talk will focus on the positive aspects of collaboration and competition in the field of exoplanet science. I take the terms "collaboration" and "competition" in their broadest senses, encompassing interactions between individual groups, agencies, countries, measurement techniques, and different observatory facilities on the ground or in space.

The history of the search for other planets is the story of competition and collaboration. A short recap of the discovery of Neptune illustrates this point [14]. In September 1845, the British mathematician and astronomer John Couch Adams communicated his preliminary calculations on the position of the perturber that was upsetting the orbit of Uranus to Cambridge Observatory Director James Challis and Astronomer Royal George Airy. Challis was unimpressed by the calculations and did nothing. The prediction languished, untested. A year later, the French mathematician Urbain Le Verrier published his prediction of the position of the perturbing body. Airy, hearing of this confirmation of Adams's result, initiated observations at Cambridge. Meanwhile, French astronomers contacted by Le Verrier showed no interest in following up his predictions. So in September 1846, Le Verrier contacted Johann Galle at the Berlin Observatory who, on his first night of observation after receipt of Le Verrier's letter, observed Neptune within 1 degree of the predicted position (and 12 degrees away, it later turned out, from Adams's prediction). Arguments over precedence for the prediction and subsequent discovery resulted in more than 30 years of bitterness between French and British astronomers.

In this story we see the competition between British and French theoreticians, the goad of competition awakening interest in the British scientific establishment, and the cooperation between a French theoretician and a German observer. Plus ça change, plus c'est la même chose. The modern search for exoplanets offers many examples where compe-

CP1158, Exoplanets and Disks: Their Formation and Diversity,

Proceedings of the Intemational Conference, edited by T. Usuda, M. Ishii, and M. Tamura

C) 2009 American Institute of Physics 978-0-7354-0695-7/09/\$25.00 
tition drives innovation and collaboration expands opportunities. The steady advance in the precision of radial velocity (RV) measurements is an excellent case in point with initial discoveries of $51 \mathrm{Peg}$ and other planets being made at the $10 \mathrm{~s}$ of $\mathrm{m} \mathrm{s}^{-1}$ level $[27,24]$ to the present day where observers routinely achieve $1-2 \mathrm{~m} \mathrm{~s}^{-1}$ accuracy $[22,15]$. On the drawing boards are still more precise measuring engines with a laser comb reference [20] potentially capable of $\mathrm{cm} \mathrm{s}^{-1}$ accuracy and new spectro-interferometers with infrared or multi-object capability $[21,13]$. The cooperation between radial velocity and transit observers highlights the importance of working together to come up with striking new results [11], e.g. determining the density of a transiting planet from its mass (RV) and radius (transit).

However, as projects become larger than individual groups or even countries can reasonably afford, collaboration becomes a necessity. We have many wonderful examples of such collaborations: the Hubble and James Webb Space Telescopes; the Akari, Herschel and Planck infrared missions; and the ALMA millimeter array. These large projects, plus smaller joint efforts on instruments or sharing of telescope time, provide encouraging examples of collaboration enabling our most ambitious endeavours.

Finally, I note the importance of the third "C", "Coordination", which must mediate between collaboration and competition. Conferences like this one at the scientist-toscientist level and high level meetings between funding agencies help to ensure that scarce resources are applied thoughtfully to address key scientific questions. The Terrestrial Planet Finder (TPF) program has had a decade of such meetings to make sure that appropriate intermediate steps are taken toward our goals of detecting other Earths and searching for life. The discussions are less about individual projects and more about making sure the goals are well defined and the technology efforts well planned so that when a major mission is executed, the world's resources are carefully allocated.

\section{COMPETITION BETWEEN GROUND AND SPACE}

In a perfect world there would be little competition between ground and space efforts. The expense of a space project is so great and its timescale for implementation so long that one should always adhere to the adage that "If it can be done from the ground, it will be done from the ground long before it can be done from space". A scientific question important enough to merit a billion dollars or more on a space project can always attract enough ground-based resources for an adequate solution in the 10 to 15 years it takes to develop a space mission. The project might not be done as well or as cleanly as from space, but the major result will be understood if a ground-based attack

can be mounted. Thus, it is critical to identify the domains uniquely suited to ground and space. I discuss this concept in the context of two areas of exoplanet research: indirect and direct detection of gas giant and terrestrial planets.

\section{Indirect Evidence for Earth-like Planets in the Habitable Zone}

The trade-off between ground and space is under intense review at the present time as the scientific community weighs the importance of an astrometric mission similar to the 


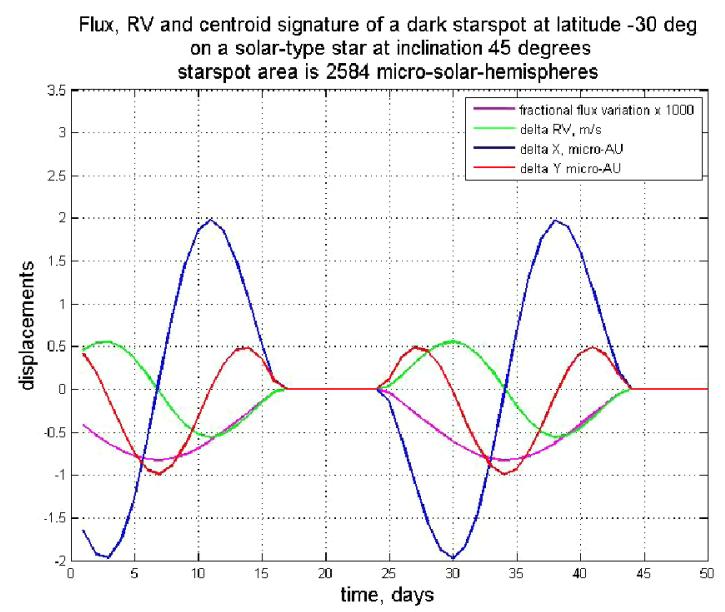

FIGURE 1. A simple model of a starspot traversing the face of a star predicts roughly $0.5 \mathrm{~m} \mathrm{~s}^{-1}$ of radial velocity noise $(1 \sigma)$ and $0.25 \mu$ as $(1 \sigma)$ of astrometric jitter for a star at $10 \mathrm{pc}$. Starting at the extreme left, from bottom to top the curves are: $\Delta \mathrm{X}$ (AU, blue), $1000 \times(\Delta F$ lux /Flux, purple), $\Delta \mathrm{Y}$ (AU, red), $\Delta \mathrm{RV}$ $\left(\mathrm{m} \mathrm{s}^{-1}\right.$, green). Courtesy J. Catanzarite.

TABLE 1. Radial Velocity and Astrometric Searches for EarthAnalogs

\begin{tabular}{lrr}
\hline & Astrometry & Radial Velocity \\
Starspot Noise $(1 \sigma, \tau=2$ week $)$ & $0.25(\mu \mathrm{as})$ & $0.5\left(\mathrm{~m} \mathrm{~s}^{-1}\right)$ \\
Earth/Sun Analog at $10 \mathrm{pc}$ & $0.3(\mu \mathrm{as})$ & $0.09\left(\mathrm{~m} \mathrm{~s}^{-1}\right)$ \\
\# Epochs for SNR $=5.8$ & 40 & 1,000 \\
Duration & $1 \mathrm{yr}$ & $40 \mathrm{yrs}$ \\
\hline
\end{tabular}

Space Interferometer Mission (SIM-Lite; Unwin et al. 2008) to find potentially habitable terrestrial planets $\left(1-5 \mathrm{M}_{\oplus}\right)$ orbiting nearby solar-type stars. These planets will someday be the targets of direct imaging systems that will look for markers of an atmosphere $\left(\mathrm{CO}_{2}\right.$ and $\mathrm{H}_{2} \mathrm{O}$ ) and even of primitive life itself (such as $\mathrm{O}_{3}$ and $\mathrm{O}_{2}$; Beichman et al. 2007). As mentioned above, the radial velocity technique has made great strides in sensitivity and is close to breaching the $1 \mathrm{~m} \mathrm{~s}^{-1}$ precision barrier. Will this be enough to reach habitable terrestrial planets or will another technique such as astrometry be needed?

The RV signature of a $1 \mathrm{M}_{\oplus}$ planet orbiting a $\mathrm{G}$ star at $1 \mathrm{AU}$ is $0.09 \mathrm{~m} \mathrm{~s}^{-1}$, independent of distance to the star. The comparable astrometric signature for a star at $10 \mathrm{pc}$ is 0.3 $\mu$ as. While RV instrumental sensitivity is improving rapidly, it is becoming apparent that the limit to RV precision is not instrumental (given access to enough time on large telescopes) but the stars themselves.

Consider a starspot covering approximately $0.1 \%$ of the solar hemisphere, a typical value for the Sun Depending on the orientation with respect to the line of sight, such a spot would cause roughly a $0.5 \mathrm{~m} \mathrm{~s}^{-1}$ variation in the measured Doppler velocity and a $0.25 \mu$ as variation in position for a star at $10 \mathrm{pc}$ (Figure 1). The effects, of 
TABLE 2. Nominal yield for $40 \%$ of SIM Lite devoted to exoplanet survey

\begin{tabular}{lccc}
\hline Mass Sensitivity at & & & \\
mid-habitable zone & $1 \mathrm{M}_{\odot}$ & $2 \mathrm{M}_{\odot}$ & $3 \mathrm{M}_{\odot}$ \\
Number of Targets Surveyed & 69 & 160 & 259 \\
\hline
\end{tabular}

course, are more complex with granulation and other photospheric phenomena being particularly important for RV observations, which depend on the measurement of line profiles. Astrometric observations are made in white light and are immune to some of these effects. This simple analysis is confirmed by careful analysis of RV measurements for stars without planets which indicate that this majority of stars (perhaps more than $80 \%$ ) have RV "jitter" as large as $1-3 \mathrm{~m} \mathrm{~s}^{-1}$ [15]. The CoRoT satellite will shortly provide data to address whether the majority of dwarf stars are as noisy or noisier than the sun $[1,5]$.

To average a $\sigma=0.5 \mathrm{~m} \mathrm{~s}^{-1}$ single measurement accuracy down to the $S=0.09 / S N R$ $\mathrm{cm} \mathrm{s}^{-1}$ precision needed for accurate detection (Signal to Noise Ratio, SNR $=5.8$; Traub et al. 2009) would require a duration of $(\mathrm{SNR} \times \sigma / S)^{2} \times \tau$ or more than 40 years, where $\tau \sim 2$ week is the coherence time of the noise source, or roughly the average lifetime of a starspot (Table 1). A comparable analysis is more encouraging for astrometric detections [36]. The comparable single measurement accuracy is $\sigma=1 \mu$ as with a stellar jitter of $<0.05 \mu$ as. Averaging down the instrumental noise to achieve $\mathrm{SNR}=5.8$ on an Earth analog orbiting a G star at $10 \mathrm{pc}$ would take only one year. More detailed examinations of the RV vs. astrometric comparison are now underway, but the conclusion is becoming clear that for the vast majority of stars which are as active or more active than the sun, $\mathrm{RV}$ jitter will preclude the detection of habitable zone earths except, perhaps, for M stars. Space astrometry with SIM-Lite accuracy will be a necessity to achieve this goal. Table 2 indicates that by using $40 \%$ of the available mission time, SIM-Lite could measure between 70 and 260 stars to the precision needed to find $1-3 \mathrm{M}_{\oplus}$ planets in the habitable zones of their parent stars.

\section{IMAGING PLANETS DIRECTLY}

\section{Observing Planets from the Ground}

The number of directly imaged planets has more than doubled within the past six months, with four objects being detected around two nearby, young A stars. Because the three planets around HR8799 [26] and the single planet around Fomalhaut [16] are young, their internal reservoirs of gravitational energy generate enough luminosity to make the objects visible [31]. Stars older than about $100 \mathrm{Myr}$ soon fade into obscurity and by $1 \mathrm{Gyr}$ are invisible with existing coronagraphic capabilities. These young planets, plus two earlier discoveries, 2M1207 [8] and GQ Lup [28], are confirmed to be companions via their common proper motion with their host star and in the case of Fomalhaut-b by orbital motion as well. What remains controversial, however, is the identification of these objects as planets ( $<13 \mathrm{M}_{J u p}$, the deuterium burning limit), as opposed to brown 
dwarfs $\left(13<M<70 \mathrm{M}_{J u p}\right)$ or even low-mass stars $\left(>70 \mathrm{M}_{J u p}\right)$. The relations between near-IR brightness, age, and mass are quite uncertain, and dynamical mass determinations are impractical for objects on long-period orbits. In fact, the models for young stars have been called into direct question. Marley et al. (2007) argued that core accretion models predict brightness levels 5-30 times lower at a given age than models that simply follow the luminosity evolution of a pre-existing ball of gas. What is missing to resolve this controversy are objects of known age for which a combination of imaging (giving luminosity, effective temperature) plus dynamical information (giving mass) is available to anchor the models. These combined data may become available with a combination of imaging using interferometers (Keck-I or VLT-I), coronagraphic imaging with ground-based telescopes or JWST, and dynamical mass measurements from ground-based RV or space-based astrometry using SIM-Lite [3, 33].

Contrast ratio levels detectable with adaptive optics on 5-10 $\mathrm{m}$ telescopes are approaching $10^{-4}-10^{-5}$ at $1^{\prime \prime}$ which corresponds to tens of $\mathrm{AU}$ for nearby young stars. There are prospects for 1-2 orders of magnitude improvement in limiting contrast over the next few years as new instruments such as the Gemini Planet Imager (GPI; Macintosh et al. 2007), P1640 at Palomar (Oppenheimer and Hinckley 2009) and Sphere for the VLT come into operation. With coronagraphs on extremely large, diffraction limited telescopes (30-42 m), it should be possible to image young (10-100 Myr), gas-giant planets orbiting within 2-3 AU of the closest young stars $(25-50 \mathrm{pc})$, and possibly even detect mature planets orbiting the nearest, low mass stars $(<5 \mathrm{pc})$ where the contrast ratio is favorable, e.g. GL 876 and GL 3522. See Figure 2 and the discussion below.

\section{Observing Giant Planets with JWST}

While JWST has a diameter of "only" $6.5 \mathrm{~m}$ compared with existing 8-10 $\mathrm{m}$ telescopes and planned 30-42 $\mathrm{m}$ telescopes on the ground, and while the $J W S T$ 's wavefront error is relatively coarse, $\sim 130 \mathrm{~nm}$, compared with the wavefront errors $<50 \mathrm{~nm}$ possible with extreme AO systems on the ground, JWST is a cooled telescope operated in an extremely stable space environment. $J W S T$ will have enormous sensitivity at exactly the wavelengths where young planets are predicted to be very bright, i.e. at $4-5 \mu \mathrm{m}$ where the transparency of their atmospheres allows radiation from hot interior levels to emerge $[2,6,7]$. The three imaging instruments on $J W S T$ each have a coronagraphic capability: NIRCam has a traditional Lyot coronagraph [18] operating from $2-5 \mu \mathrm{m}$; the Canadian Tunable Filter Imager [9] has a traditional Lyot coronagraph plus an innovative nonredundant mask imaging capability at 3-5 $\mu \mathrm{m}$ [32]; the mid-IR instrument, MIRI [30] has four quadrant phase masks operating around $10 \mu \mathrm{m} . J W S T$ should be able to observe planets more massive than $0.1 \mathrm{M}_{J u p}$ outside $1^{\prime \prime}$ with its Lyot coronagraphs and planets more massive than $1 \mathrm{M}_{\text {Jup }}$ inside $1^{\prime \prime}$ with its NRM interferometric mode.

Figure 2 and Figure 3 show the fractional yield of a ground-based instrument (P1640 at Palomar) and a space-based instrument (the NIRCAM coronagraph on $J W S T$ ) surveying a sample of 650 young stars (Beichman et al. 2009, in preparation). In this Monte Carlo simulation, planets of various masses $\left(0.1-40 \mathrm{M}_{J u p}\right)$ were placed at distances between 0.5-200 AU from the star. The brightness of the planet was taken from models 


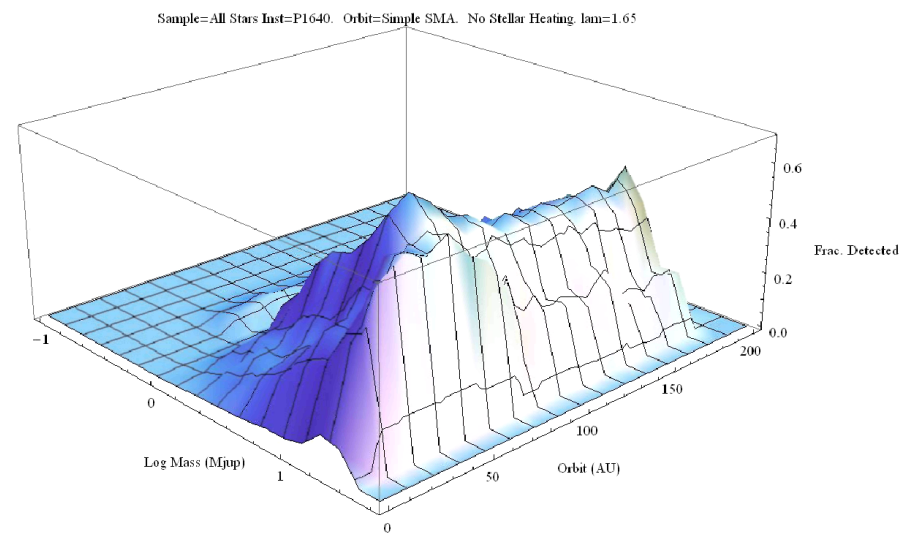

FIGURE 2. A Monte Carlo simulation shows the fraction of planets of a given mass and age orbiting a sample of 650 young stars that were detectable using the Lyot coronagraph on the P1640 instrument at $1.6 \mu \mathrm{m}$.

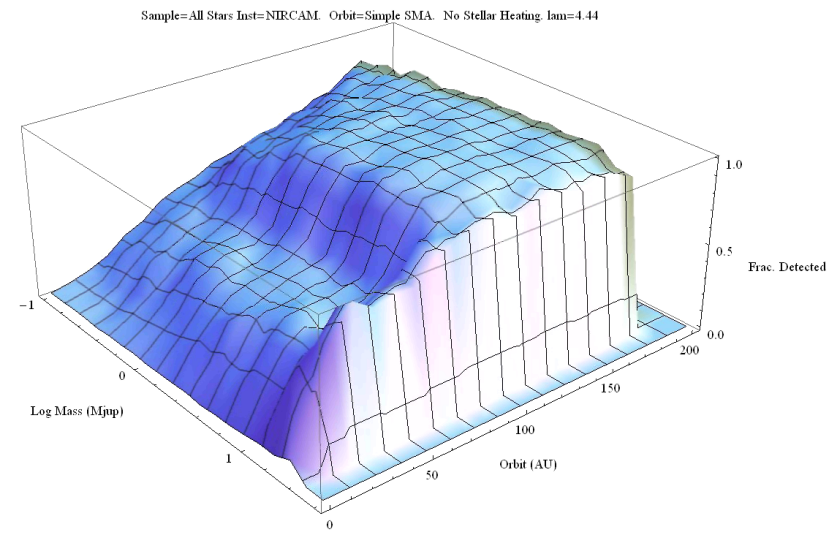

FIGURE 3. A Monte Carlo simulation shows the fraction of planets of a given mass and age orbiting a sample of 650 young stars that were detectable using the Lyot coronagraph on JWST's NIRCam instrument at $4.4 \mu \mathrm{m}$.

appropriate to the planetAfs mass and the age of the host star [2]. The average planet detected by JWST has a mass of $2 \mathrm{M}_{J u p}$ with an age of $70 \mathrm{Myr}$ and located at $130 \mathrm{AU}$. There is a long tail of detections of planets with masses as low as $0.1 \mathrm{M}_{J u p}$ for the closest stars. Comparable values for the ground-based search with P1640 at Palomar is an average mass of $8 \mathrm{M}_{J u p}$ with an age of $10 \mathrm{Myr}$ and located at $110 \mathrm{AU}$. There is a long tail of detections for planets within than $50 \mathrm{AU}$ for the closest stars. Detection of these planets will test the efficancy of disk fragmentation mechanisms for the formation of gas giant planets [10]. Spectroscopy of these systems will help to assess their physical properties. A separate analysis shows that JWST, but not ground-based telescopes, will 
be able to find 1-2 Gyr, $1 \mathrm{M}_{\text {Jup }}$ gas giants around the nearest $M$ stars.

\section{Imaging Terrestrial Planets}

The previous section demonstrates that both ground-based facilities and JWST will be challenged to study young, gas giant planets. Neither is capable of the much more demanding task of detecting and characterizing Earth analogs orbiting nearby stars. While transit observations may enable direct detection of some "super Earths" ( $\sim 2$ $\mathrm{R}_{\oplus}$ ) orbiting $\mathrm{M}$ stars $[12,17]$, the general task of direct imaging will require space telescopes of exquisite precision: an ultra-high contrast coronagraph operating on a $>4 \mathrm{~m}$ visible light telescope, a nulling interferometer operating over a $50-100 \mathrm{~m}$, baseline in the mid-infrared, or a $50 \mathrm{~m}$ diameter occulter operating tens of thousands of $\mathrm{km}$ in front of a $4 \mathrm{~m}$ telescope (TPF-C, DARWIN/TPF-I, or TPF-O, respectively). For details, the reader is referred to the proceedings of a recent conference (http: //exep.jpl.nasa.gov/exep \_exForum. cfm) and community report [19] on exoplanet missions.

\section{CONCLUSIONS}

Competition, collaboration and coordination form the cornerstones of progress in most human endeavors. Exoplanet research is no exception. Competition serves as a spur to innovation and rapid progress. Collaboration serves to allocate intellectual and financial resources efficiently for projects larger than what a small group can comfortably undertake. And coordination through frequent meetings such as these ensures an appropriate balance between competition and collaboration. This philosophy has brought us great successes, from HST to Herschel/Planck, and promises to to so in the future with JWST and ALMA. In the long term, we hope that many of the participants of this conference will help implement a mission that will find and characterize Earth analogs and search for life on other worlds.

\section{ACKNOWLEDGMENTS}

We have benefited from discussion about $S I M$ with Joe Catanzerite. Isabelle Baraffe and Travis Barman generously adapted their models to the JWST filter passbands. Ben Oppenheimer, Anand Sivaramakrishnan and Sasha Hinkley provided useful discussions about the performance of P1640 and the non-redudant mask on JWST. We gratefully acknowledge travel support from the organizers and thank our Japanese hosts for their commitment to international collaboration in ground and space astronomy over the years. Some of the research described in this publication was carried out at the Jet Propulsion Laboratory, California Institute of Technology, under a contract with the National Aeronautics and Space Administration. Finally, I would like to thank Ben Oppenheimer, Dave Latham and Dimitar Sasselov for their hospitality during my sabbatical leave. 


\section{REFERENCES}

1. Affer, L., Micela, G. Favata, R., et al. 2009, in Cool Stars, Stellar Systems and the Sun: Proceedings of the 15th Cambridge Workshop on Cool Stars, Stellar Systems and the Sun, AIP Conf. Proceedings, $1094,341$.

2. Baraffe, I., Chabrier, G., Barman, T. S., et al. 2003, $A \& A, 402,701$.

3. Beichman, C. A. 2001 in Young Stars Near Earth: Progress and Prospects, ASP Conf. Series Vol. 244. Edited by R. Jayawardhana and T. Greene. San Francisco: Astronomical Society of the Pacific, 376.

4. Beichman, C.A., Fridlund, M., Traub, W. A., et al. 2007 in Protostars and Planets V, B. Reipurth, D. Jewitt, and K. Keil (eds.), University of Arizona Press, Tucson, 915.

5. Bonomo, A.S. and Lanza, A.F. 2009, $A \& A, 482,341$.

6. Burrows, A., Sudarsky, D. and Lunine, J. I. 2003, ApJ, 596, 587.

7. Burrows, A., Budaj, J., and Hubeny, I. 2008, $A p J, 678,1436$.

8. Chauvin, G. et al. 2005, $A \& A, 438, \mathrm{~L} 29$.

9. Beaulieu, M., Doyon, R. and Lafrenière, D. 2008, Proceedings of the SPIE, Edited by Oschmann, J. M., Jr; de Graauw, M.W. M.; MacEwen, H. A., 7010, 70103J-70103J.

10. Boss, A. $2000, A p J, 545$, L61.

11. Charbonneau, D. 2008, in Proceedings of IAU 253: Transiting Planets, eds, F. Pont et al., Cambridge Univ. Press, in press.

12. Deming, D. et al. 2009, eprint arXiv:0903.4880.

13. Ge, J. 2009, Bull AAS, \#213, \#336.02.

14. Grosser, M. 1979 The Discovery of Neptune, Dover Press, ISBN-13: 978-0486237268.

15. Howard, A. et al. 2009, ApJ, 696, 75.

16. Kalas, P. et al. 2008, Science, 322, 1345.

17. Kaltenegger, L. and Traub, W. A. 2009, eprint arXiv:0903.3371.

18. Krist, J. 2007, in In the Spirit of Bernard Lyot: The Direct Detection of Planets and Circumstellar Disks in the 21st Century. University of California, Berkeley, CA, USA. Edited by Paul Kalas.

19. Lawson, P. R. Traub, W. A. and Unwin, S. R. http://exep.jpl.nasa.gov/documents/ExoplanetCommunityReport.pdf

20. Li, Chih-Hao et al. 2008, Nature, 452, 610.

21. Lloyd, J., et al. 2009, Transiting Planets, Proceedings of the International Astronomical Union, IAU Symposium, 253, 157.

22. Lovis et al. 2003, Nature, 441, 305 .

23. Macintosh, B. and the GPI Consortium 2007, in In the Spirit of Bernard Lyot: The Direct Detection of Planets and Circumstellar Disks in the 21st Century, University of California, Berkeley, CA, USA. Edited by Paul Kalas.

24. Marcy, G. W. and Butler, R. P. Bull AAS, 27, 1379.

25. Marley, M. S., Fortney, J. J., Hubickyj, O., et al. 2007, ApJ, 655, 541.

26. Marois, C. et al. 2008, Science, 322, 1348.

27. Mayor, M. and Queloz, D. 1995, Nature, 378, 355.

28. Neuhäuser, R., Guenther, E. W., Wuchterl, G., et al. 2005, $A \& A$, 435, L13.

29. Oppenheimer, B. and Hinckley, S. 2009, Ann. Rev. Astr. Astrop., in press.

30. Rouan, D., Boccaletti, A., Baudoz, P., et al. 2007, in In the Spirit of Bernard Lyot: The Direct Detection of Planets and Circumstellar Disks in the 21st Century, University of California, Berkeley, CA, USA. Edited by Paul Kalas.

31. Saumon, D., Hubbard, W. B., Burrows, A., et al. 1996, ApJ, 460, 993.

32. Sivaramakrishnan, A., Tuthill, P., Ireland, M., et al. 2009, American Astronomical Society, AAS Meeting \#213, \#350.03.

33. Tanner, A. et al. 2007, $P A S P, 119,747$.

34. Traub, W.A. et al. 2009, arXiv:0904.0822.

35. Unwin et al. 2008, PASP, $120,38$.

36. Unwin, S. C., Catanzarite, J., Shao, M. 2009, American Astronomical Society, DDA meeting \#40, $\# 17.05$. 